\title{
Evaluation of Disinfection Quality of Dental Faculty Units of Ahvaz Jundishapur University of Medical Sciences, Southwest of Iran in 2017
}

\author{
Seyed Morteza Bagheri ${ }^{1}$, Mohammad Shooriabi², Mansour Amin ${ }^{3}$, Fatemeh Babadi ${ }^{4}$, Asadollah Ahmadzadeh ${ }^{5}$, \\ Mahsan sabet ${ }^{6} \&$ Hamid Reza Mozaffari ${ }^{7}$ \\ ${ }^{1}$ Department of Pediatric Dentistry, Faculty of Dentistry, Tabriz Branch, Islamic Azad University, Tabriz, Iran \\ ${ }^{2}$ Department of Oral Medicine, School of Dentistry, Ahvaz Jundishapur University of Medical Sciences, Ahvaz, \\ Iran \\ ${ }^{3}$ Health Research Institute, Infectious and Tropical Diseases Research Center and Department of Microbiology, \\ School of Medicine, Ahvaz Jundishapur University of Medical Sciences, Ahvaz, Iran \\ ${ }^{4}$ Department of Oral and Maxillofacial Medicine, School of Dentistry, Ahvaz Jundishapur University of Medical \\ Sciences, Ahvaz, Iran \\ ${ }^{5}$ Department of Prosthodontics, School of Dentistry, Ahvaz Jundishapur University of Medical Sciences, Ahvaz, \\ Iran \\ ${ }^{6}$ School of Dentistry, Ahvaz Jundishapur University of Medical Sciences, Ahvaz, Iran \\ ${ }^{7}$ Department of Oral and Maxillofacial Medicine, School of Dentistry, Kermanshah University of Medical \\ Sciences, Kermanshah, Iran \\ Correspondence: Mohammad Shooriabi, Department of Oral Medicine, School of Dentistry, Ahvaz Jundishapur \\ University of Medical Sciences, Ahvaz, Iran. E-mail: dsshoriabii@yahoo.com
}

Received: November 30, 2017

Accepted: January 2, 2018

Online Published: July 23, 2018

doi:10.5539/jmbr.v8n1p88

URL: https://doi.org/10.5539/jmbr.v8n1p88

\begin{abstract}
Introduction: Performing dental practices in the oral environment leads to the transmission of microorganisms in saliva and blood to working surfaces and dental devices and their infection. Preventing transmission of infection through these devices is an important task of a dentist. Hence, this research was conducted to evaluate the disinfection quality of the dental faculty units of Ahvaz Jundishapur University of Medical Sciences (AJUMS) in Iran.
\end{abstract}

Materials and Methods: In order to evaluate the quality of disinfection of the units, sampling was performed from all glasses spittoon surfaces of 90 units of the clinical unit of the AJUMS dental faculty before and after disinfection by personnel. Then, the bacteria were cultured in a medium and examined.

Results: The mean (and standard deviation) of the total infection of units of the dental faculty was 46534.4 (583380.4) colonies per $1 \mathrm{ml}$ before disinfection and 40265.6 (52131.1) colonies per $1 \mathrm{ml}$ after disinfection, reflecting significant decrease in number of bacterial colonies after disinfection $(\mathrm{P}<0.001)$. In addition, a significant decrease was seen in the number of bacterial colonies in the restoration, pediatric, orthodontic and diagnosis units $(\mathrm{p}<0.05)$, but the difference before and after disinfection was not significant in the prosthetic, endodontic, surgical and periodontal units. In addition, the most common types of microorganisms in the whole units of the dental faculty were pseudomonas aeruginosa, Staphylococcus aureus and Streptococcus, respectively, and after disinfection, the most common types of microorganisms were Pseudomonas aeruginosa, Staphylococcus aureus and Enterococcus, respectively.

Conclusion: In general, this study showed that the disinfection method of units in dental faculty can not reduce the severity of infection of the units. Given what was stated, it is recommended that the method and the substances used to disinfect the unit to be changed.

Keywords: infection control, disinfection methods, Deconex, dental faculty 


\section{Introduction}

Performing dental actions in the oral environment transmits the microorganisms in saliva and blood to working surfaces, dental devices and instruments and their infection. Thus, preventing the transmission of infection through these devices is an important task of a dentist (Javaheri \& Zanganeh, 2008; Valian, Shahbazi, Farshidnia, \& Tabatabaee, 2014). In most dental treatments, infection with blood is unavoidable. Thus, steps should be taken to eliminate the microbes accurately from the devices used in dentistry (Taheri, Bakianian, Fallah, \& Nik Kerdar, 2010; Eskandarloo \& Mashouf, 2006). Although the devices should be sterilized daily with dry heat and autoclave, there is a need for devices and equipment which cannot be sterilized by thermal methods such as unit chair, rotary, unit glasses spittoon, cabinets, amalgamators, external surfaces of oven, suction, floor and walls (Sultanpour \& Sayed, 2001). Generally, release of suspended particles in the dental environment is one of the ways of transmission of infection in dentistry. Clinical surfaces in dentistry are not directly in contact with the patient, although they can be infected during work and then act as a source of microbial infection. These surfaces can be infected directly through the diffusion of suspended particles and splashing blood, saliva and water containing body fluids or through contact with infected dental devices or contact with infected gloves . The light key, the unit key, the control key of chair and dental stool are examples of clinical surfaces (Valian, Shahbazi, Farshidnia, \& Tabatabaee, 2014). Although the use of disposable covers on some surfaces touched by the dentist has been accepted, some parts of the unit often remain uncovered and the principles of infection control are ignored in these parts. Dental stool or dental chair is one of the cases prone to infections due to proximity to the unit and the site for performing the dental practice. Experience has shown that most students and dentists often replace the dental stool with infected gloves, and thereby, they indirectly transmit the infection (Teksoy et al., 2011; Cristina et al., 2008). The unit glasses spittoon is also continuously infected by blood and saliva. Therefore, these surfaces should be disinfected to prevent the transmission of infection. Control of pathogenic agents in the environment requires the use of appropriate methods for disinfection. Disinfection is performed by physical and chemical agents. By using them, we can reduce the rate of microorganisms causing pathogens. Chemicals such as $70 \%$ Alcohol and deconex, used as solvent in the workplace, are some of these substances (Teksoy et al., 2011). The performance of disinfectants will be effective if used in the manner recommended by the manufacturing company (Christensen et al., 1989; Best et al., 1990; Ardakani et al., 2008). Inappropriate use will not result in proper cleaning and disinfection. Thus, the type of its using by the consumer will affect the result of the work. Based on the reports of different studies on the dental unit infection (Valian, Shahbazi, Farshidnia, \& Tabatabaee, 2014; Taheri, Bakianian, Fallah, \& Nik Kerdar, 2010; Sultanpour \& Sayed, 2001; Cristina et al., 2008; Ardakani et al., 2008), this study was conducted to evaluate the disinfection quality of dental units at Ahvaz Jundishapur University of Medical Sciences (AJUMS).

\section{Methods and Materials}

In this study, in order to evaluate the quality of disinfection of units, sampling was performed from all glasses spittoon surfaces of 90 clinical units of Ahvaz Jundishapur University of Medical Sciences (AJUMS) Dental faculty including pediatric, prosthetic, endodontic, restoration, periodontal, orthodontic and surgical units. In addition, defective units and the units, which were not used, were excluded from study. Sampling was performed before disinfection of the unit at the end of the working day, at 14:45. Then, the service force, which is responsible for disinfecting the units, disinfected the units in accordance with the procedure. The sample taken after disinfection of the units was taken on the morning of the next day and at the beginning of the working day, at 8:00 am (about sixteen hours later). All parts of the unit glasses spittoon were sampled by a sterilized swab in a width of $2 \mathrm{~cm}$ and a length of $10 \mathrm{~cm}$, which was immersed to sterilized physiology serum. The swap movement on the blood agar was rotary. The swab was then placed in a sterile test tube containing $5 \mathrm{ml}$ of sterile physiology serum and the test tube was closed. Samples were immediately transferred to the laboratory of microbiology during 10 minutes at the Faculty of Medicine at the clod box (manufactured by Igloo Company of USA). The same thing was done in the same way after disinfection of the unit by the personnel exactly from the place where it was first sampled. Sterile gloves were used during sampling and in each time of sampling, the gloves were replaced. In the laboratory, test tubes containing the sample were shaken for 30 seconds to isolate the bacteria completely, and in the vicinity of the flame at a radius of $15-20 \mathrm{~cm}, 0.01 \mathrm{cc}$ of the sample was taken by sampler and placed in blood agar culture media. The culture medium was incubated for 24 hours at $37^{\circ} \mathrm{C}$ and in $10 \%$ co 2 conditions. Then, the culture medium was removed from heating room and the number of colonies was counted. To obtain the colony count of bacteria in $1 \mathrm{cc}$ of physiological serum, the number of obtained colonies was multiplied by 100 . In order to identify the dominant bacterium, one of the most similar colonies was cultured in blood agar culture medium and incubated for 24 hours at $37{ }^{\circ} \mathrm{C}$ and conditions of $10 \% \mathrm{co} 2$. Then, for isolation of bacteria Gram positive and gram negative, cocci and Bacillus bacteria, gram staining lam was prepared. Then, each of bacteria was tested for 
the diagnostic tests according to the shape below the microscope. Biochemical tests such as catalase, coagulase to detect gram-positive cocci, other tests such as oxidase, nitrate, and citrate, fermentation of lactose sugars, galactose, glucose and sucrose were used for differential diagnosis of other bacteria. To examine the samples before and after disinfection of the unit, McNeimar and the descriptive statistics analysis were used, which it was performed in SPSS 22 software.

\section{Results}

The findings of this study showed that the mean (and standard deviation) of the total units of the dental faculty was 46534.4 colonies per $1 \mathrm{ml}$ before disinfection and 40265.6 colonies per $1 \mathrm{ml}$ after disinfection, indicating that the infection of units decreased by 6269 clooneies on average after the disinfection. This decrease was statistically significant $(\mathrm{P}<0.001)$ (Table 1). The findings also showed that the most common types of microorganisms in the whole units of the dental faculty before the disinfection were Pseudomonas aeruginosa (17\%), Staphylococcus aureus (12\%) and Streptococcus spp (11\%), respectively . The most common types of microorganisms in the whole units of the dental faculty after disinfection were Pseudomonas aeruginosa (32\%), Staphylococcus aureus (11\%), and Enterococcus (10\%) (Table 2). In addition, bacterial infection in each unit before and after disinfection was also studied in this study. The results of this study are presented in Table 3.

Table 1. Infection of units before and after disinfection of the environment in the whole dental faculty

\begin{tabular}{cccc}
\hline Colony per $\mathbf{1}$ ml & Before disinfection & After disinfection & P.value \\
\hline Number of units & 90 & 90 & \\
mean & 46534.4 & 40265.6 & 0.001 \\
SD & 53380.4 & 52131.1 & \\
\hline
\end{tabular}

Table 2. Frequency distribution of the most common types of microorganisms in the whole unit of the dental faculty

\begin{tabular}{lcccc}
\hline \multirow{2}{*}{ types of microorganisms } & \multicolumn{2}{c}{ Before disinfection } & \multicolumn{2}{c}{ after disinfection } \\
\cline { 2 - 5 } & $\mathrm{f}$ & $\%$ & $\mathrm{f}$ & $\%$ \\
\hline Pseudomonas aeruginosa & 15 & 17 & 29 & 32 \\
Staphylococcus aureus & 11 & 12 & 10 & 11 \\
Streptococcus spp & 10 & 11 & 9 & 10 \\
Enterococci & - & - & 9 & 10 \\
Total & 90 & 100 & 90 & 100 \\
\hline
\end{tabular}

Table 3. Comparison of the mean number of bacterial colonies in each unit of the dental faculty before and after disinfection

\begin{tabular}{|c|c|c|c|c|c|c|}
\hline \multirow[t]{2}{*}{ unit } & \multirow[t]{2}{*}{$\mathbf{n}$} & \multicolumn{2}{|c|}{ before disinfection } & \multicolumn{2}{|c|}{ after disinfection } & \multirow[t]{2}{*}{ p-value } \\
\hline & & mean & SD & mean & $\mathrm{SD}$ & \\
\hline Diagnosis & 8 & 687.5 & 581.71 & 250.00 & 261.86 & $* 0.011$ \\
\hline Orthodontic & 12 & 1958.33 & 1962.12 & 983.33 & 860.06 & $* 0.05$ \\
\hline Pediatric & 13 & 17661.33 & 40984.34 & 1238.46 & 2729.63 & $* 0.002$ \\
\hline Restoration & 9 & 52030.8 & 55897.1 & 35684.6 & 61620.9 & $* 0.012$ \\
\hline Periodontic & 9 & 61366.67 & 57672.43 & 49444.44 & 57456.94 & 0.342 \\
\hline Prosthesis & 12 & 101500.00 & 29444.86 & 101333.33 & 30022.21 & 0.317 \\
\hline Endodontic & 10 & 57150.00 & 55800.9 & 56740.00 & 56200.46 & 0.124 \\
\hline Surgical & 13 & 70100.00 & 52651.9 & 69361.54 & 5355.98 & 0.134 \\
\hline Total & 86 & 48554.65 & 53760.66 & 42031.4 & 52671.55 & $* * 0.000$ \\
\hline
\end{tabular}

*significant difference at the level of 0.05

$* *$ significant difference at the level of 0.001

\section{Discussion}

Despite all of its dangers for health of dentists, dentistry is one of the popular fields of study in Iran (12-16). As patients use a unit glasses spittoon in most of dental treatments to wash their mouths and discharge the accumulated fluids in the mouth, and this area is not covered with plastic or other disposable materials, infection with blood 
and saliva of this area is unavoidable. On the other hand, when using the unit glasses spittoon, there is a possibility of contact of this area with the patient's hands and face (especially in children). Therefore, elimination of microbe from this area seems to be an essential (Valian, Shahbazi, Farshidnia, \& Tabatabaee, 2014; Cristina et al., 2008). In this regard, various methods have been developed in recent years for disinfection of equipment and various surfaces in the dental office and various studies have been carried out in this regard. Given the different results of the studies (Christensen et al., 1989; Best et al., 1990; Ardakani et al., 2008; Larsen et al., 2000; Azimi-Hoseini et al., 2007) on the effect of these methods, this study was conducted to evaluate the quality of disinfection of dental units of the Ahvaz Jundishapur University of Medical Sciences (AJUMS) in southwest of Iran in 2017. It should be noted that in the pilot study of this study, it was found that the disinfection of the units was performed by the service forces and the solution used to disinfect the surfaces and units of the dental faculty of Ahvaz was DESOMED RAPID AF (Dr. Trippen co, German). This solution is quaternary ammonium compound containing alcohol and was one of the intermediate design facts. In a study conducted to investigate the antibacterial effect of three disinfectant solutions on different levels of dentistry in Iran, Nosuhi et al. (2012) compared the Deconex, Microzed and Alprocid solutions with each other. The findings of this study showed significant difference between the number of bacterial colonies before and after disinfection and any of the three disinfectant solutions (Nasoohi et al., 2012). Anbari et al. (2014) also showed that ethanol, oxygenated water and Deconex, compared to ultraviolet radiation, had an acceptable disinfection effect on the surface and equipment of the research laboratory and showed that Deconex reduced the microbial load (Anbari, Nabi, \& Khalili, 2014). The present study also showed that the use of DESOMED RAPID AF (Dr. Trippen co, German) reduced the total mean microbial load of the evaluated area in units of dental faculty after disinfection, and this decrease was statistically significant $(p<0.001)$. These findings are consistent with those of previous studies.

As the mean number of bacterial colonies in the total units examined to show the effects of disinfecting solution can be influenced by standard deviation and as in each treatment unit, the type of dental services provided to patients and thus the use of unit glasses spittoon and the rate and type of infection created in different units are different, for more accurately evaluation of the result of the disinfection process, the effect of DESOMED RAPID on the microbial infection was also investigated separately in each unit. Interestingly, the results showed that a decrease in the number of bacterial colonies was observed in restoration, pediatric, orthodontic and diagnosis units, and there was a significant difference in the level of infection in these units before and after disinfection. There was no significant difference before and after disinfection in the prosthetic, endodontic, surgical and Periodontic units. This could be due to the difference in treatment provided in these units. The presence of any infection in at least one plate, even fewer than 10 colonies per $1 \mathrm{cc}$, represents a lack of sterilization. Presence of at least 100 colonies per plate has been defined as severe infection. It has also been observed that there is no clear difference between the 100-colonial boundary in 1 cc solution and higher boundaries (Azimi-Hoseini et al., 2007). Thus, severe infection is defined as presence of more than 100 colonies per $1 \mathrm{cc}$, unfortunately, it is concluded that the disinfection process in the faculty has not been effective in practice and the rate of infection in the units before and after disinfection, despite significant reduction in the number of colonies in the culture of samples in some units, is high.

In justifying these results, it can be stated that aerosols with a diameter of less than 50 microns can remain in the suspended space for a long time (Rautemaa, Nordberg, Wuolijoki-Saaristo, \& Meurman, 2006; Coleman, O'Donnell, Boyle, \& Russell, 2010) and after that the disinfection process perfumed at the end of the day, they sat during fourteen to fifteen hours on different parts of the unit, including the unit glasses spittoon and caused infection and positive microbial culture. Accordingly, it is recommended that the unit disinfection process to be performed before working on each patient in order to provide more safety, especially for people who have a weakened immune system. Infecting endodontic files with salivary of 30 patients admitted to the restorative, endodontic and surgical units, Sabouri et al examined the disinfecting effects of microton and deconex. They identified the following microbes in infected files: Staphylococcus aureus, Nonpathogenic, Staphylococcus, Streptococcus Viridans pneumonias, Neisseriae suprophyticus, Corynebacterium spp, Candida albicans and Bacillus subtilis (Saboori, Fallah, \& Dastgerdi, 2006). In examining the effects of deconex, microton, cidex for disinfection of turbines and parasites in the restorative unit at Shiraz Dental Faculty in Iran Sharafeddin et al also isolated the following microbes: Viridans streptococci, Diphtheroidase, Filamentous fungi, various species of Veillonella and Lactobacillus (Sharafadin, Sadeghi, \& Kohanteb, 2004). In the present study, the most common isolated bacteria included Pseudomonas aeruginosa, Staphylococcus aureus, Streptococcus and Enterococcus. These results indicate that isolated microbial species vary widely in different studies. These differences may be due to the fact that a specific therapeutic unit was considered in each study and the method of research was also very different in these studies. 
Al-Hiyasat et al in Iran showed that the presence of Pseudomonas aeruginosa in $86 \%$ of the water systems of dental units at the beginning of the working day. They showed that the number of pseudomonas aeruginosa colonies decreased after working with unit compared to that in beginning of work (Al - Hiyasat, Ma'ayeh, Hindiyeh, \& Khader, 2007). In the present study, the most common microbial strain cultured before and after the disinfection of unit was Pseudomonas aeruginosa. Moreover, the number of colonies not only did not decrease one day after disinfection, but also increased. In this regard, the results of these two studies are consistent. The reason for this increase in the number of colonies is the proliferation of Pseudomonas aeruginosa microbe during one day with the help of organic particles present in the biofilm on the unit glasses spittoon (24). This bacterium also prevents the growth of other bacteria by producing bacteriocin. The use of unit during the day by applying water pressure leads to elimination of bacteria, which have a slight adhesion to biofilm. Pseudomonas aeruginosa is a gramnegative opportunistic pathogenic bacterium, so that the rate of mortality in those affected with Pseudomonas aeruginosa has been reported 50\%. One of the characteristics of Pseudomonas aeruginosa is high resistance to the types of antibiotics (Poole, 2004; Estahbanati, Kashani, \& Ghanaatpisheh, 2002; Ebrahimzadeh Shiraz, Rezaei Yazdi, \& Alijanianzadeh, 2017). Schelstraete et al showed that patients with cystic fibrosis had received Pseudomonas aeruginosa out of the home environment (such as the dental environment) (Schelstraete et al., 2007). Therefore, the transmission of this pathogen to patients with weakened immune system in dental centers is a great concern. According to some researchers, the effects of disinfection of quaternary ammonium compounds, such as Deconex or DESOMED RAPID AF (Dr. Trippen co, German), are reduced against hard water and organic and non-organic sediments such as gas and cotton (Saboori, Fallah, \& Dastgerdi, 2006; American Dental Association, 1992; Oeding, 2004). It seems that when these items are taken into account when disinfecting the units, the effectiveness of the disinfectant increases. However, in the study conducted by Azimi Hosseini et al in Iran, none of the disinfectants (Deconex and Microton) were able to completely eliminate the Pseudomonas aeruginosa (Azimi-Hoseini et al., 2007). Considering this issue and the results of this study, it seems that the use of another disinfectant such as hypochlorite besides the DESOMED RAPID solution to be necessary to overcome this bacterium. In addition, the issue of knowledge management should also be considered in this regard, and the special attention should be paid to storing, sharing and applying the results of this research (providing the right information to right people at the right time) (Gilavand, 2017).

\section{Conclusion}

In general, this study showed that the disinfection methods in dental faculties cannot change the severity of infection of the units. The most common microbial strain in the units of the faculty was Pseudomonas aeruginosa, which is an opportunistic bacterium. It can cause disease in patients with weakened immune system. The used solution cannot eliminate this bacterium. Given what was reported, it is recommended that the method and the type of substance used for disinfecting to be changed.

\section{Acknowledgement}

This research was adopted from a thesis written by Mahsan sabet, MD student of Dentistry in Ahvaz Jundishapur University of Medical Sciences, numbered GP95201 and the ethical code of IR.AJUMS.REC.1396.127

\section{References}

Al-Hiyasat, A. S., Ma'ayeh, S. Y., Hindiyeh, M. Y., \& Khader, Y. S. (2007). The presence of Pseudomonas aeruginosa in the dental unit waterline systems of teaching clinics. International journal of dental hygiene, 5(1), 36-44.

American Dental Association. (1992). Infection control recommendations for the dental office and the dental laboratory. J Am Dent Assoc, 1-8.

Anbari, F., Nabi, A., \& Khalili, M. (2014). Comparison of 70\% alcohol, Deconex, H2O2 and UV Light for disinfection of surfaces and equipment's infertility Research laboratory of Yazd. TKJ., 6(3), 12-19. (In Persian).

Ardakani, F. E., Zandi, H., Mohammadi, Z., Ayatollahi, J., Ayatollahi, F., \& Behniafar, B. (2008). Comparing the Disinfecting Efficacies of Micro 10, Deconex, Alprocid and Microzid AF on the Microorganisms on radiographic equipments. Journal of dental research, dental clinics, dental prospects, 2(2), 48-52.

Azimi-Hoseini, S., Shahcheraghi, F., Ghaemmaghami, A., Sheikholeslamian, S. M., \& Mousavi Azadkasmaei, P. (2007). The clinical efficacy of QAC (micro 10 and Deconex 53 Plus) on contaminated dental instruments. The Journal of Islamic Dental Association of Iran, 19(2), 35-41. (In Persian).

Best, M., Sattar, S. A., Springthorpe, V. S., \& Kennedy, M. E. (1990). Efficacies of selected disinfectants against Mycobacterium tuberculosis. Journal of Clinical Microbiology, 28(10), 2234-2239. 
Christensen, R. P., Robison, R. A., Robinson, D. F., Ploeger, B. J., Leavitt, R. W., \& Bodily, H. L. (1989). Antimicrobial activity of environmental surface disinfectants in the absence and presence of bioburden. The Journal of the American Dental Association, 119(4), 493-505.

Coleman, D. C., O'Donnell, M. J., Boyle, M., \& Russell, R. (2010). Microbial biofilm control within the dental clinic: reducing multiple risks. Journal of Infection Prevention, 11(6), 192-198.

Cristina, M. L., Spagnolo, A. M., Sartini, M., Dallera, M., Ottria, G., Lombardi, R., \& Perdelli, F. (2008). Evaluation of the risk of infection through exposure to aerosols and spatters in dentistry. American journal of infection control, 36(4), 304-307.

Ebrahimzadeh Shiraz, T., Rezaei Yazdi, H., \& Alijanianzadeh, M. (2017). Evaluation of Carbapenemase resistance in Pseudomonas aeruginosa and Enterobacteriaceae family isolated from clinical specimens by using phenotypic methods. Pars Journal of Medical Sciences, 14(4), 9-15. (In Persian).

Eskandarloo, A., \& Mashouf, R. Y. (2006). The Evaluation of Bacterial Contamination of Active Radiography Apparatus in Dental Centers of Hamadan City. Scientific Journal of Hamadan University of Medical Sciences, 12(4), 55-59. (In Persian).

Estahbanati, H. K., Kashani, P. P., \& Ghanaatpisheh, F. (2002). Frequency of Pseudomonas aeruginosa serotypes in burn wound infections and their resistance to antibiotics. Burns, 28(4), 340-348.

Gilavand, A. (2017). The comparison of the tuition-paid and free tuition dental students' incentives in choosing their field of study at Ahvaz Jundishapur University of Medical Sciences, Southwest of Iran. Annals of Tropical Medicine and Public Health, 10(5), 1254-1259. doi: 10.4103/ATMPH.ATMPH_316_16.

Gilavand, A. (2016). The Comparison of Iranian and Foreign Students' Motivations to Choose Dentistry Field of Study. International Journal of Pediatrics, 4(6), 1993-2010. doi: 10.22038/ijp.2016.6861

Gilavand, A. (2017). A Study of Ahvaz Jundishapur University of Medical Sciences Dental Students' Interest to Pursuing a Specialty According to Their Demographic Information. Indo American Journal of Pharmaceutical Sciences, 4(6), 1593-1598. doi.org/10.5281/zenodo.819543.

Gilavand, A. (2017). Examining the Knowledge Management Status in Iranian Universities: A Review, Indo Am. JP Sci, 4(11), 4219-4225. https://doi.org/10.5281/zenodo

Gilavand, A., \& Espidkar, F. (2018). EVALUATING THE ACADEMIC STATUS AND JOB PROSPECT OF DENTISTRY GRADUATES IN IRAN. INDO AMERICAN JOURNAL OF PHARMACEUTICAL SCIENCES, 5(5), 3520-3524. doi.org/10.5281/zenodo.1243214

Gilavand, A., \& Shooriabi, M. (2016). Investigating the Relationship between Mental Health and Academic Achievement of Dental Students of Ahvaz Jundishapur University of Medical Sciences. International Journal of Medical Research \& Health Sciences, 5(7S), 328-333.

Javaheri, M, \& Zanganeh, N. (2008). Evaluating antibacterial effects of three disinfectants on dental operatory surfaces. J Qazvin Univ Med Sci., 11(4), 36-41. (In Persian).

Larsen, T., Fiehn, N. E., Peutzfeldt, A., \& Owal, B. C. (2000). Disinfection of dental impressions and occlusal record s by ultra V Raditition. Prosthodent Restor Dent, 8(2), 77-4.

Nasoohi, N., Vand Yousefi, J., Mahdisear, F., \& Sheikhiekh, G. Z. M. (2012). Evaluation of Antibacterial Effects Of Three Disinfectant Solutions On Dental Operatory Surfaces. J Res Dent Sci., 9(1), 36-43. (in Persian).

Oeding, M. (2004). Infection control. Retrieved from WWW.Fice.com.pdf/re.pdf.

Poole, K. (2004). Efflux-mediated multiresistance in Gram-negative bacteria. Clinical Microbiology and infection, $10(1), 12-26$.

Rautemaa, R., Nordberg, A., Wuolijoki-Saaristo, K., \& Meurman, J. H. (2006). Bacterial aerosols in dental practice-a potential hospital infection problem?. Journal of hospital infection, 64(1), 76-81.

Saboori, A., Fallah, F., \& Dastgerdi, M. A. (2006). Comparison on two disinfectants: Micro10+ and Deconex 53 plus on dental instruments. The Journal of Islamic Dental Association of IRAN (JIDA), 18(1), 49-55. (In Persian).

Schelstraete, P., De Boeck, K., Proesmans, M., Lebecque, P., Leclercq-Foucart, J., Malfroot, A., ... \& De Baets, F. (2007). Pseudomonas aeruginosa in the home environment of newly infected cystic fibrosis patients. European Respiratory Journal. 
Sharafadin, F., Sadeghi, A. R., \& Kohanteb, G. (2004). Compression of the effect of deconex (solarsept), micro 10 and sidexin disinfecting dental instrument. Shiraz Uni Dent J, 6(1-2), 38-41. (In Persian).

Sultanpour, J., \& Sayed, S. A. (2001). Evaluation of the quality and spectrum of microtechnical solutions. Islamic Dental Association of Iran, 13(3), 7-21. (In Persian).

Taheri, J., Bakianian, V. P., Fallah, F., \& Nik Kerdar, N. (2010). Assessment of the efficacy of BIB forte in disinfection of dental instruments. J Dent Sch., 27(4), 179-186. (In Persian).

Teksoy, A., Alkan, U., Eleren, S. Ç., Topaç, B. Ş., Şağban, F. O. T., \& Başkaya, H. S. (2011). Comparison of indicator bacteria inactivation by the ultraviolet and the ultraviolet/hydrogen peroxide disinfection processes in humic waters. Journal of water and health, 9(4), 659-669.

Valian, A., Shahbazi, R., Farshidnia, S., \& Tabatabaee, F. S. (2014). Evaluation of the bacterial contamination of dental units in restorative and peridontics departments of Dental School of Shahid Beheshti University of Medical Sciences. J Mash Dent Sch, 37(4), 345-56. (In Persian).

\section{Copyrights}

Copyright for this article is retained by the author(s), with first publication rights granted to the journal.

This is an open-access article distributed under the terms and conditions of the Creative Commons Attribution license (http://creativecommons.org/licenses/by/4.0/). 